\title{
Revista Colombiana de

\section{Infección de injerto carótido-carotídeo para debranching aórtico: tratamiento con injerto autológo de vena}

\section{César Eduardo Jiménez ${ }^{\mathrm{a}, \mathrm{b}, \mathrm{c}, *}$, Leonardo Randial ${ }^{\mathrm{d}}$, Mabel Facundo $^{\mathrm{d}}$ y Fernando Quiroga ${ }^{\mathrm{c}}$}

\author{
a Servicio de Cirugía Vascular, Hospital Universitario Clínica de San Rafael, Bogotá, Colombia \\ b CINTHEV- Centro Integral de Heridas y Enfermedades Vasculares, Bogotá, Colombia \\ c Fundación Sinergia y Sociedad, Chía, Colombia \\ ${ }^{\mathrm{d}}$ Hospital Universitario Clínica San Rafael, Bogotá, Colombia
}

Recibido el 13 de julio de 2017; aceptado el 22 de marzo de 2018

Disponible en Internet el 1 de junio de 2018

\section{PALABRAS CLAVE \\ Cirugía híbrida; \\ Vasos; \\ Aneurisma complejo}

\section{KEYWORDS}

Hybrid surgery;

Vessels;

Complex aneurysm
Resumen El uso de prótesis vasculares y dispositivos endovasculares ha permitido tratar muchas patologías que antes no se intervenían dado el gran riesgo quirúrgico, como es el caso de los aneurismas de aorta torácica. Los materiales usados para estas reconstrucciones son muy resistentes y de larga duración, pero son muy sensibles a ser colonizados y presentar infección, lo que genera morbimortalidad considerable.

Se presenta el caso de un paciente de 64 años con antecedente de aneurisma del arco aórtico y aorta descendente, tratado en forma extra institucional con prótesis de aorta torácica endovascular y debranchig de las ramas del arco aórtico, con injerto carótido-carotídeo y carótido-subclavio izquierdo en dacrón, por vía pretraqueal, con antecedente de intento de cubrimiento del injerto expuesto con un flap miocutáneo el cual fue fallido por necrosis; ingresó al Hospital Universitario Clínica de San Rafael, con exposición del injerto carótido-carotídeo a nivel cervical, signos locales de infección y sepsis secundaria.

(c) 2018 Sociedad Colombiana de Cardiología y Cirugía Cardiovascular. Publicado por Elsevier España, S.L.U. Este es un artículo Open Access bajo la licencia CC BY-NC-ND (http:// creativecommons.org/licenses/by-nc-nd/4.0/).

Infection of a carotid-carotid graft for aortic debranching: treatment with an autologous venous graft

Abstract The use of vascular replacements and endovascular devices has led to the treatment of many conditions that could not be operated on due to a high surgical risk, such as aortic aneurysms. The materials used for these reconstructions are very resistant and durable, but are

\footnotetext{
* Autor para correspondencia.

Correo electrónico: cesarejmd@yahoo.com (C.E. Jiménez).
} 
very susceptible to being colonised and infected, which leads to a considerable morbidity and mortality.

The case is presented of a 64 year-old patient with a history of an aortic arch and descending aortic aneurysm. This had been treated in another hospital with a thoracic aortic endovascular repair and debranching of the aortic arch, with carotid-carotid and left carotid-subclavian Dacron graft. The pretracheal route was used, after attempting to cover the exposed graft with a myocutaneous flap that failed due to necrosis. The patient was admitted to the Hospital Universitario Clínica de San Rafael, presenting with a carotid-carotid graft at cervical level, with local signs of infection and secondary sepsis.

(c) 2018 Sociedad Colombiana de Cardiología y Cirugía Cardiovascular. Published by Elsevier España, S.L.U. This is an open access article under the CC BY-NC-ND license (http:// creativecommons.org/licenses/by-nc-nd/4.0/).

\section{Introducción}

El uso de prótesis vasculares y dispositivos endovasculares ha permitido tratar muchas patologías que antes no se intervenían por riesgo quirúrgico altísimo, como es el caso de los aneurismas de aorta torácica. Los materiales usados para estas reconstrucciones son muy resistentes y de larga duración, pero son muy sensibles a ser colonizados y presentar infección, lo que genera muy alta morbimortalidad ${ }^{1,2}$. La infección protésica se trata con resección o explantación, procedimientos que pueden generar sangrado, falla multiorgánica, sepsis o isquemias tisulares. La incidencia de infección para prótesis vasculares y endovasculares es del 0,5 al $5 \%$ y se asocia con la indicación de la intervención, patologías asociadas del paciente, sitio de implantación y técnica quirúrgica.

La infección protésica luego de cirugía de la aorta torácica es mucho menor que la cirugía de la aorta abdominal o procedimientos periféricos, y mucho menor en casos de procedimientos endovasculares, y oscila entre 0,5 y $1 \%^{3,4}$.

Se presenta el caso de un paciente de 64 años con antecedente de aneurisma del arco aórtico y aorta descendente, manejado de manera extrainstitucional con una prótesis de aorta torácica endovascular y un debranching de las ramas del arco aórtico, con injerto carótido-carotídeo y carótidosubclavio izquierdo en dacrón, por vía pretraqueal, con antecedente de intento de cubrimiento del injerto expuesto con un flap miocutáneo el cual fue fallido por necrosis, quien ingresó al Hospital Universitario Clínica de San Rafael, con exposición del injerto carótido-carotídeo a nivel cervical (fig. 1), signos locales de infección y sepsis secundaria.

AngioTAC de tórax: prótesis endovascular aórtica desde la porción distal del ostium de la arteria carótida común izquierda hasta tercio distal de la aorta descendente sin fugas ni signos de infección; injerto carótido-carotídeo y carótido-subclavio permeable con gas a su alrededor. Leucocitosis de 21.000 con desviación izquierda y PCR (proteína c reactiva) $230 \mathrm{mg} / \mathrm{l}$.

Análisis: paciente con sepsis secundaria a infección de injerto de dacrón, expuesto en cuello, en el que se intentó cubrimiento con flap miocutáneo, que fue fallido; requiere explantación del material protésico y reemplazo con material autológo, de otra manera la sepsis no se controlará y el riesgo de sangrado masivo y muerte es muy alto.

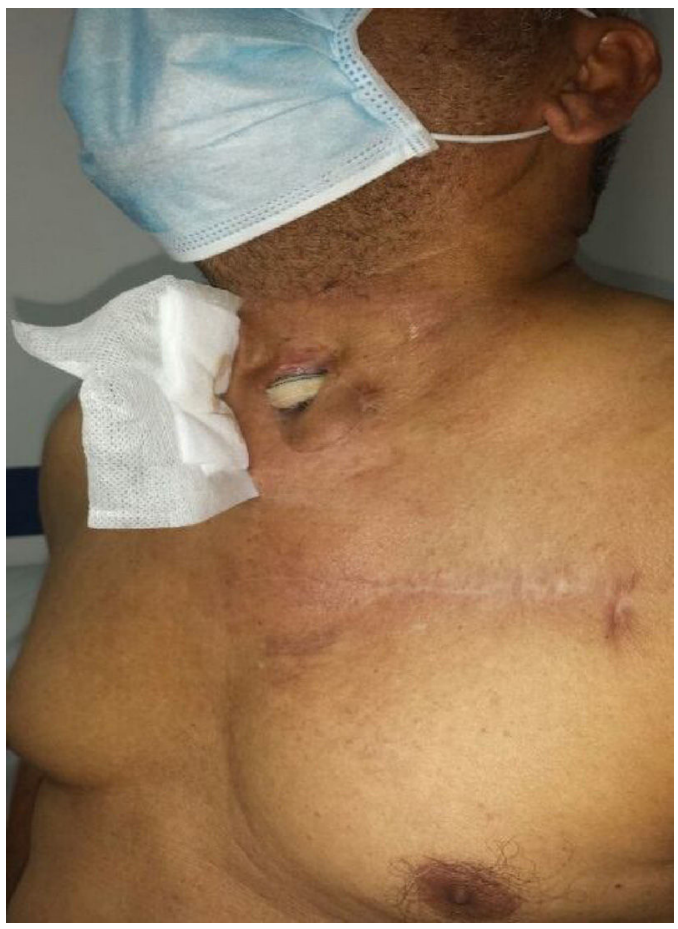

Figura 1 Exposición de injerto vascular infectado en cuello.

Técnica quirúrgica: se realizó una esternotomía mediana convencional para control de los vasos supraaórticos, más cervicotomía antero-lateral bilateral; se disecó el tronco braquiocefálico y la aorta ascendente; se procedió a disección de arterias carótidas comunes de manera bilateral, encontrando injerto carótido-carotídeo en dacrón severamente infectado con pus a su alrededor pero permeable, e injerto carótido-subclavio izquierdo en continuidad en dacrón en iguales condiciones de infección. Ambos injertos ubicados en el compartimento anterior del cuello, vía pretraqueal (fig. 2).

Se procedió a disección cuidadosa de las estructuras comprometidas y a toma de injerto venoso, de vena femoral derecha con técnica de Clagget, preservando la vena safena mayor y su cayado. Se preparó una extensión de injerto de $10 \mathrm{~cm}$ y se procedió a resección completa del injerto carótido-carotídeo y carótido-subclavio y se colocó 


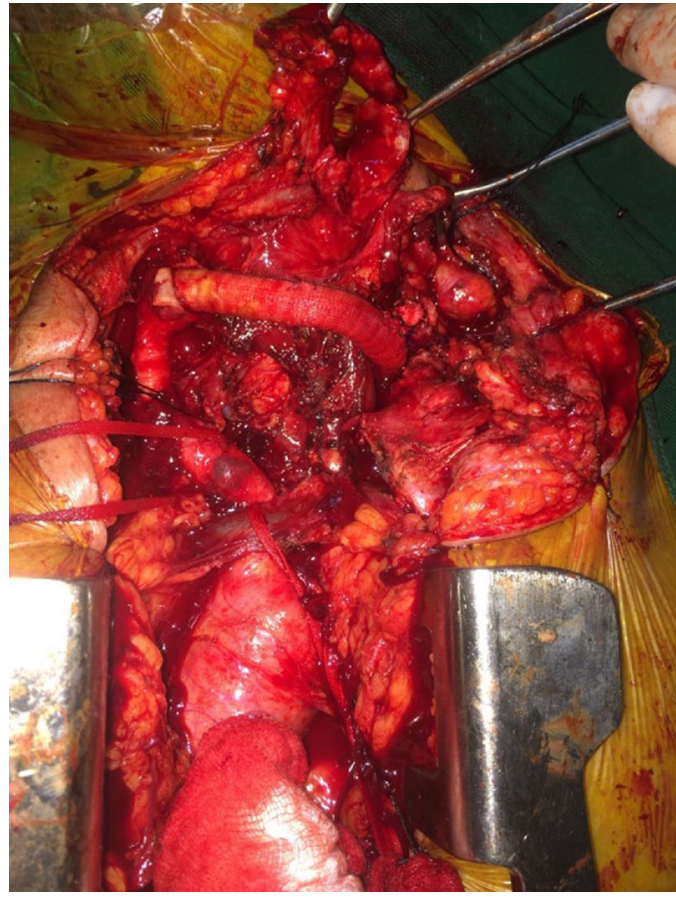

Figura 2 Esternotomía con disección de troncos supraaórticos y presencia de injerto de dacrón infectado pretraqueal.

un nuevo injerto en vena femoral autóloga por vía retrofaríngea (fig. 3); se resecó el injerto carótido subclavio, ligando la arteria subclavia izquierda y rafia a la arteria carótida común.

Se lavó profusamente la zona quirúrgica, se cerraron las incisiones y se colocaron drenajes. El paciente evolucionó satisfactoriamente, con estancia en $\mathrm{UCl}$ de 3 días,

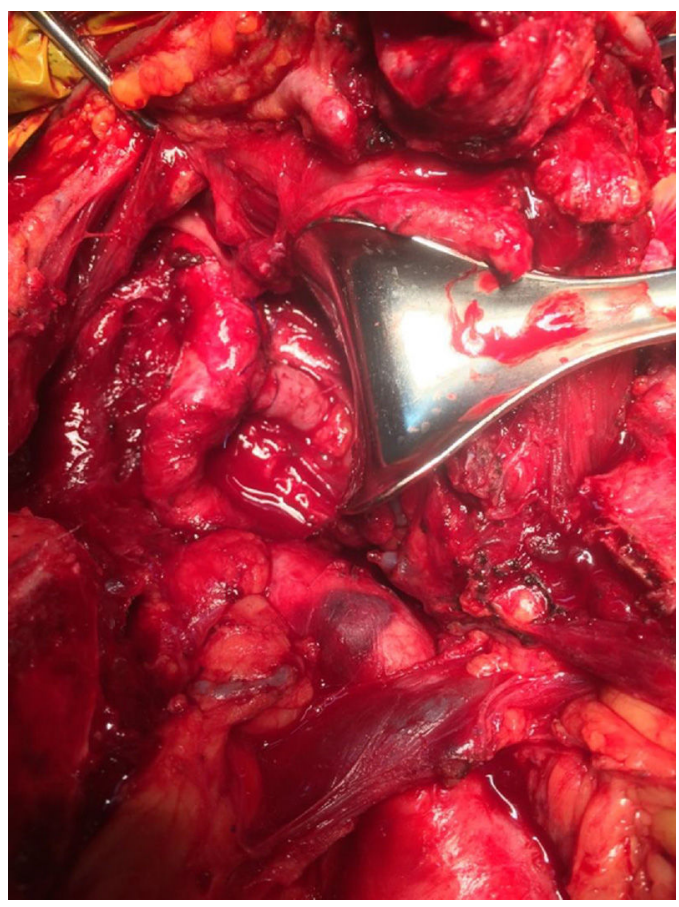

Figura 3 Construcción de injerto carótido-carotídeo en vena autóloga femoral por vía retrofaríngea. lográndose controlar el proceso séptico; se dio manejo antibiótico intrahospitalario por 12 días y se dio de alta a los 14 días de hospitalización, para manejo antibiótico por 6 semanas con ertapenem.

$\mathrm{Ha}$ estado en seguimiento durante 9 meses, tiempo durante el cual se ha observado buena evolución, ausencia de signos de infección y permeabilidad de las reconstrucciones arteriales sin lesiones neurológicas centrales o periféricas.

\section{Discusión}

El tratamiento endovascular de los aneurismas de aorta torácica, fue descrito por primera vez en 1994, por el grupo de Dake et al. En 2005, la FDA aprobó el primer dispositivo a partir de lo cual se han hecho millones de implantes alrededor del mundo para patologías como: aneurismas, disección, hematoma intramural, úlcera aórtica penetrante, transección aórtica traumática, fístula traqueoesogáfica, entre otras. Se ha demostrado, por tanto, en muchos estudios, que ya no está indicada la cirugía abierta para estas patologías, en vista de que la mortalidad es menor en la cirugía endovascular, que en la abierta $(2,1$ vs. $11,7 \%)$, y disminuye el índice de complicaciones, paraplejia (3 vs. 14\%), falla ventilatoria (4 vs. $20 \%$ ) y falla renal $(1 \text { vs. } 13 \%)^{4-6}$. La cirugía abierta convencional del arco aórtico requiere el uso de circulación extracorpórea e hipotermia y tiene una mortalidad que alcanza el 10 al $20 \%$, de modo que las técnicas endovasculares e híbridas tienen un papel preponderante ${ }^{7}$.

La introducción y evolución de las técnicas endovasculares y quirúrgicas para las patologías de aorta torácica ha contribuido a aumentar las indicaciones de intervención y a disminuir los índices de morbimortalidad. Al mezclar procedimientos quirúrgicos y endovasculares al tiempo o en estadios, se crean los procedimientos híbridos como debranching o relocalización, que consiste en retirar los ostium de salida de estas arterias y relocalizarlos en una zona más proximal del arco o incluso la aorta ascendente, de tal manera que se puedan implantar así prótesis de manera más proximal.

El concepto de cirugía híbrida para el arco aórtico, tiene tres principios básicos ${ }^{8,9}$ :

1. Relocalización quirúrgica abierta de los grandes vasos del arco aórtico.

2. Creación de una zona de aterrizaje o anclaje adecuada para una prótesis endovascular.

3. Despliegue concomitante de una prótesis endovascular de aorta torácica.

Este tipo de procedimientos ya ha demostrado ser eficaz en el manejo de aneurismas del arco aórtico y aorta descendente. Existen diferentes técnicas, que de manera acumulada, muestran una mortalidad del $5,8 \%$, evento cerebrovascular del $2,8 \%$ e isquemia espinal del $2,9 \%^{7,8}$. No hay estadísticas asociadas a la infección de estas reconstrucciones extraanatómicas, a excepción de algunos reportes de caso, en los que se tratan con cirugía de explantación o prótesis endovasculares periféricas como el Viahban (Goremedical, Flagstaff, AZ, USA) ${ }^{9}$. En general, la indicación en estos casos es hacer la derivación por vía retrofaríngea, 
ya que se disminuye el riesgo de infección y es más anatómica. La mortalidad del injerto carótido-carotídeo y la incidencia de evento cerebrovascular intraoperatorio es de menos del $5 \%$; patencia primaria del $88 \%$ y secundaria del $92 \%$ a 3 años ${ }^{10-12}$.

Ante infección protésica o de un dispositivo endovascular, se debe individualizar cada caso y tener en cuenta los siguientes factores: presentación clínica (aguda o crónica), localización de la infección, estado general del paciente, tipo de injerto o prótesis comprometida e infección localizada o sistémica. La exploración quirúrgica permite inspeccionar el injerto, tomar muestras, evaluar tejidos adyacentes y controlar sangrados. La mayoría de casos se descubren por aparición de pus en las heridas, y en pocos se presenta estallido del injerto con sangrado masivo, que es la complicación más temida y de mayor mortalidad en este tipo de infecciones ${ }^{11,12}$.

Los principios generales de tratamiento en estos casos, son $^{13-15}$ :

1. Resecar el material protésico infectado.

2. Mantener la continuidad arterial.

3. Resecar el tejido necrótico e infectado alrededor.

4. Instaurar antibioticoterapia prolongada, de acuerdo con los cultivos.

Las prótesis vasculares se pueden contaminar por tres factores, a saber ${ }^{16,17}$ :

1. Contaminación intraoperatoria, por falla en la técnica aséptica.

2. Infección activa que colonice la prótesis al momento del procedimiento (infección urinaria, neumonías, etc.).

3. Infección propia del aneurisma o del trombo, donde existan bacterias que proliferen al momento de la colocación del injerto y lo invadan.

En este paciente se cumplieron todos los principios, logrando resecar todo el material infectado, desbridar tejidos necróticos y preservar la continuidad arterial por medio de un injerto en vena, que es más resistente a la infección.

\section{Conclusión}

Pese al uso de antibióticos y los principios en prevención de infecciones quirúrgicas, las infecciones de prótesis vasculares continúan siendo una entidad frecuente; este caso muestra una infección compleja en una patología aneurismática de alto nivel, en cuyo caso el manejo integral con diagnóstico imagenológico y la planeación quirúrgica adecuada, genera buenos resultados y disminuye la morbimortalidad.

\section{Conflictos de intereses}

Ninguno.

\section{Bibliografía}

1. Darouiche R. Treatment of infections associated with surgical implants. N Engl J Med. 2004;350:1422-9.

2. Swain TW III, Calligaro KD, Dougherty MD. Management of infected aortic prosthetic grafts. Vasc Endovasc Surg. 2004;38:75.

3. Scharrer-Pamler R, Kotsis T, Kapfer X, Gorich J, Orend $\mathrm{KH}$, Sunder-Plassmann L. Complications after endovascular treatment of thoracic aortic aneurysms. J Endovasc Ther. 2003;10:711-8.

4. Makaroun MS1, Dillavou ED, Kee ST, Sicard G, Chaikof E, Bavaria $\mathrm{J}$, et al. Endovascular treatment of thoracic aortic aneurysms: results of the phase II multicenter trial of the GORE TAG thoracic endoprosthesis. J Vasc Surg. 2005;41:1-9.

5. Bavaria JE, Appoo JJ, Makaroun MS, Verter J, Yu ZF. Mitchell RS; Gore TAG Investigators Endovascular stent grafting versus open surgical repair of descending thoracic aortic aneurysms in low risk patients: a multicenter comparative trial. J Thorac Cardiovasc Surg. 2007;133:369-77.

6. Matsumura JS, Cambria RP, Dake MD, Moore RD, Svensson LG, Snyder S. TX2 Clinical Trial Investigators International controlled clinical trial of thoracic endovascular aneurysm repair with the Zenith TX2 endovascular graft: 1-year results. J Vasc Surg. 2008;47:247-57, discussion 257.

7. Patel V, Mukhopadhyay S, Ergul E, Aranson N, Conrad MF, Lamuraglia GM, et al. Impact of hospital volume and type on outcomes of open and endovascular repair of descending thoracic aneurysms in the United States Medicare population. J Vasc Surg. 2013;58:346-54.

8. Milewski RK, Szeto WY, Pochettino A, Moser GW, Moeller P, Bavaria JE. Have hybrid procedures replaced open aortic arch reconstruction in high-risk patients? A comparative study of elective open arch debranching with endovascular stent graft placement and conventional elective open total and distal aortic arch reconstruction. J Thorac Cardiovasc Surg. 2010;140:590-7.

9. Fadda G, Marino M, Kasemi H, Di Angelo L. Hybrid endovascular solutions for supra-aortic vessels extra-anatomic bypass infection. Ann Vascular Surg. 2015;29, 1662.e1-1662.e5.

10. Goksel O, Guven K, Karatepe C, Gok E, Acunas B, Cinar B, Alpagut $U$. Debranching solutions in endografting for complex thoracic aortic dissections. Arq Bras Cardiol. 2014;103:154-60.

11. De Rango P, Cao P, Ferrer C, Simonte G, Coscarella C, Cieri E, et al. Aortic arch debranching and thoracic endovascular repair. J Vasc Surg. 2014;59:107-14.

12. Abou-Zamzam AM, Moneta GL. Edwards JMa. Extrathoracic arterial grafts performed for carotid artery occlusive disease not amenable to endarterectomy. Arch Surg. 1999;134:952-7.

13. Irace L, Martinelli O, Stumpo R, Trenti E, Fornasin FR, Laurito A, et al. Carotid carotid bypass. Minerva Cardioangiologica. 2003;51:329-35.

14. Ozsvath KJ, Roddy SP, Darling C, Byrne J, Kreienberg PB, Choi $D$, et al. Carotid-carotid crossover bypass: is it a durable procedure? J Vasc Surg. 2003;37:582-5.

15. O'Connor S, Andrew P, Batt M, Becquemin JP. A systematic review and meta-analysis of treatments for aortic graft infection. J Vasc Surg. 2006;44:38-45.

16. Coselli JS, Kö ksoy C, LeMaire SA. Management of thoracic aortic graft infections. Ann Thorac Surg. 1999;67:1990-3.

17. LeMaire SA, Coselli JS. Options for managing infected ascending aortic grafts. J Thorac Cardiovasc Surg. 2007;134:839-43. 